\title{
MR-based Attenuation Correction for PET/MR
}

\author{
M. Hofmann ${ }^{1,2}$, F. Steinke ${ }^{1}$, I. Bezrukov ${ }^{1,2}$, A. Kolb ${ }^{2}$, P. Aschoff ${ }^{2}$, M. Lichy ${ }^{2}$, M. Erb ${ }^{2}$, T. Nägele ${ }^{2}$, M. Brady ${ }^{3}$, B. Schölkopf , and B. Pichler $^{2}$

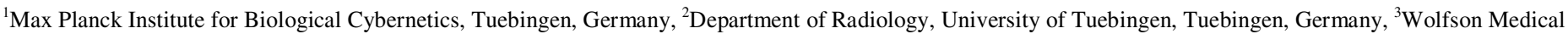 \\ Vision Laboratory, University of Oxford, Oxford, United Kingdom
}

\section{Introduction}

There has recently been a growing interest in combining PET and MR. While promising hardware prototypes have been presented [1], the software for combined imaging is in its infancy. PET attenuation correction (AC), which accounts for radiation attenuation properties of the tissue, is mandatory for quantitative PET. Usually the attenuation map in stand-alone PET or PET/CT is obtained from a transmission scan or from a CT image. In the case of PET/MR, there is insufficient space for the rotating source so that the tissue attenuation information needs to be determined from the MR image. This is inherently difficult: The PET attenuation value is not related to the proton density and tissue relaxation properties expressed in the MR intensities. For example bone compacta and air both typically do not contribute a MR signal, whereas their attenuation values are maximally distinct.

Here, we report our progress on evaluating suitable MR sequences and methodology for calculating the attenuation images ("Pseudo-CT") based on the MR image. We present results for head and whole body imaging. To our knowledge, our contribution is the first to show the use of ultra short echo time (UTE) sequences for AC in clinical examples. It is also among the first to show results for human whole body AC.

\section{Materials and Methods}

For brain imaging, we acquired at $3 \mathrm{~T}$ an ultra short echo time (UTE) MR image with TE $=0.07 \mathrm{~ms}$ and $1.1 \times 1.1 \times 1.1 \mathrm{~mm}^{3}$ resolution. Together with a standard FLASH sequence (TE=2.46ms) bone could be identified as the structure that yields signal in the UTE image, but not in the FLASH. PseudoCT images were estimated by computing class probabilities $\mathrm{p}(\mathrm{C} \mid \mathrm{I})$ for four classes and converting them into CT-intensities by weighting default attenuation values for each class with their respective probability.

In whole body imaging, there is not sufficient time for UTE acquisition, and instead, at 1.5T, we used a FLASH protocol that acquires opposed phased and inphase images in one scan. Resolution was $2.6 \times 2.6 \times 2.6 \mathrm{~mm}^{3}$ and $\mathrm{TA}=18 \mathrm{~s}$, which allowed for breath hold acquisition. The information in the two FLASH echos is, however, not sufficient for determining the tissue class. Additional information is necessary. We pursued an atlas-based approach, where the atlas comprises an MR image and a corresponding co-registered CT-image of the same subject. For a new patient, a non-rigid registration algorithm can then automatically compute a deformation field that aligns the atlas MR image with the patient's MR image. Applying the same deformation to the atlas CT image yields a "Pseudo"-CT image for the new subject. This kind of atlas based prediction relies on the assumption that it is possible to find an intersubject registration between atlas MR image and MR image of the new patient. Realising that this assumption does not always hold, we additionally employ an approach that enhances atlas registration through combination with a pattern recognition method that takes into account local MR image information [2].

\section{Results}

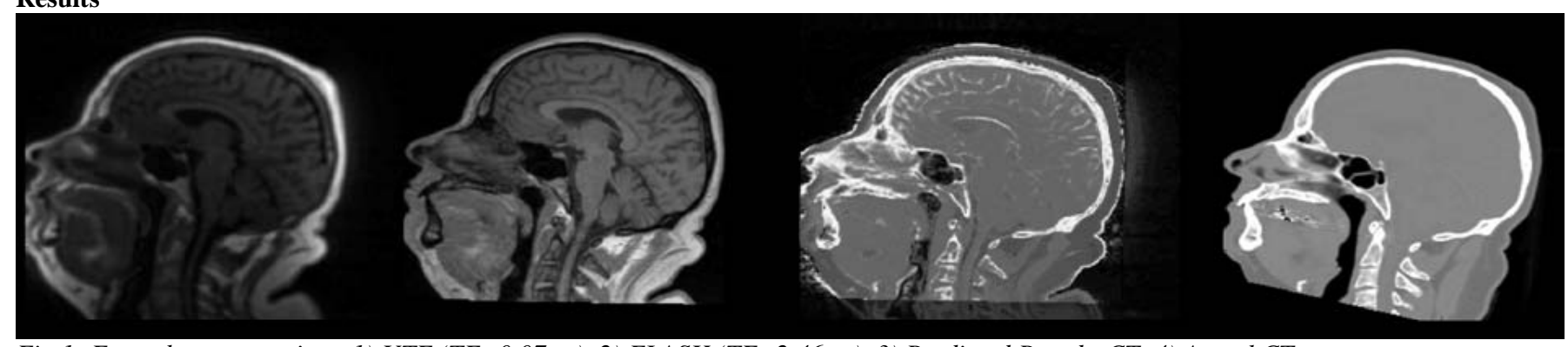

Fig 1: From the same patient: 1) UTE (TE=0.07ms) 2) FLASH (TE=2.46ms) 3) Predicted Pseudo-CT 4) Actual CT scan

Figures 1 and 2 show the Pseudo-CT images that were obtained for head and whole body, respectively. Comparison with the actual CT scans show that on average 94\% (head) and 93\% (whole body) of voxels are classified correctly among the relevant classes air/fat/soft tissue/bone.

\section{Conclusion and Next Steps}

We have presented MR sequences and image processing tools that allow the prediction of an attenuation map (Pseudo-CT). The results using our methodology are promising and data acquisition on a larger scale is currently ongoing.

\section{References}

[1] "Simultaneous PET/MRI: A new Approach for Functional and Morphological Imaging”, M.S. Judenhofer et al.; Nature Medicine 2008, 14, 459 - 465.

[2] "MRI-based Attenuation Correction for PET/MRI: A Novel Approach

Combining Pattern Recognition and Atlas Registration“, M. Hofmann, F. Steinke,

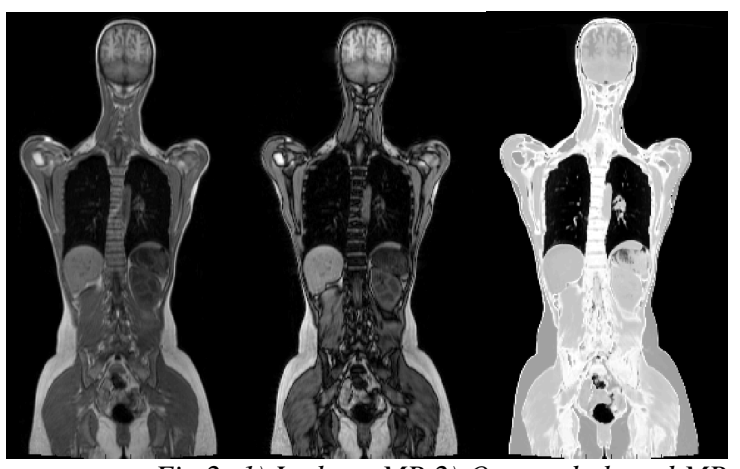

Fig 2: 1) Inphase MR 2) Opposed phased MR

3) Predicted PseudoCT

V. Scheel, G. Charpiat, J. Farquhar, P. Aschoff, M. Brady, B Schölkopf, B.J. Pichler, J Nucl Med 2008 49(11): 1875-1883 